\title{
The Global Geodetic Observing System (GGOS): Detecting the Fingerprints of Global Change in Geodetic Quantities
}

\author{
Hans-Peter Plag ${ }^{\mathrm{a}}$, Chris Rizos ${ }^{\mathrm{b}}$, Markus Rothacher ${ }^{\mathrm{c}}$, Ruth Neilan ${ }^{\mathrm{d}}$ \\ ${ }^{a}$ Nevada Bureau of Mines and Geology and Seismological Laboratory, University of Nevada, \\ Reno, Mail Stop 175, Reno, NV 89557, hpplag@unr.edu; \\ ${ }^{b}$ School of Surveying \& Spatial Information Systems, University of New South Wales, \\ Sydney NSW 2052, Australia, c.rizos@unsw.edu.au; \\ ${ }^{\mathrm{c}}$ ETH, Zürich, Switzerland \\ ${ }^{\mathrm{d}}$ NASA Jet Probulsion Laboratory, Pasadena, CA, USA
}

\begin{abstract}
Modern geodetic observations from a wide range of space and terrestrial technologies contribute to our knowledge of the solid Earth, atmosphere, ocean, cryosphere, and land water storage. These geodetic observations record the "fingerprints" of global change processes and thus are a crucial independent source of high accuracy information for many global change studies. Many of the geodetic techniques require a globally distributed ground infrastructure, and associated space segment elements. In the past decade and half a variety of technique-specific services have been established under the auspices of the International Association of Geodesy (IAG) to facilitate global coordination of geodetic activities and to ensure the generation of high accuracy and reliable geodetic products to support geoscientific research. The Global Geodetic Observing System (GGOS) is an important component of the IAG, and is intended to be an "umbrella" for the IAG Services, with a primary coordinating function to ensure the development of an adequate global geodetic infrastructure, and a suite of integrated multi-technique products, that will meet the needs of scientific users. Coordination means bringing together the different geodetic observing techniques, services and analysis methods so as to ensure that the same standards, conventions, models and parameters are used in the data analysis and modelling of "Earth system" processes. Integration implies the combination of geometric, gravimetric, and rotational observations in data analysis and data assimilation, and the joint estimation and/or modelling of all the necessary parameters representing the difference components of the Earth system. The geodetic observations collected during the last decades have facilitated major scientific discoveries related to geohazards, climate and the global water cycle. Geodesy has the potential to contribute even more to global change studies, particularly if coordination and integration of the geodetic activities are continued.
\end{abstract}

Keywords: global change, GGOS, geodesy, IAG

\section{INTRODUCTION}

Humanity is increasingly being confronted with the limitations of a restless planet, with finite resources that cannot meet growing demands and a limited capacity to accommodate the impact of the increasingly dominant anthropogenic factor. The anthroposphere has grown into one of the most powerful factors in the Earth system, which is transforming the Earth's surface layers rapidly [15], and which is capable of changing major system processes. To illustrate the scale of the impact, consider the fact that more than $50 \%$ of the ice-free surface of the solid Earth has been transformed by humans. Among experts there is a broad consensus that humanity is changing the planet's climate [5]. One could say that Earth has entered the geological epoch of the Anthropocene.

The Earth system is subject to a variety of dynamic processes driven by interior and exterior forces, operating on a wide range of temporal and spatial scales. As a consequence, large areas of the Earth's surface are exposed to natural hazards, including geohazards, storms, storm surges, and floods. Urban settlements are sprawling into areas at high risk from such natural hazards, thus increasing the vulnerability of society. At the same time, 
natural and anthropogenic climate change, is changing the hazards and introducing a large uncertainty into any predictions of future events and developments. Considering the scale of the anthropogenic impact on the Earth system, it is clear that decisions made today will influence the well-being of future generations. Yet our limited capability of predicting the consequences of our activities, including effectiveness of different mitigation strategies, hampers the development of mitigation and adaptation policies.

A number of recent World Summits have emphasized the paramount importance of sustainable development for a prosperous future of the anthroposphere and confirmed the necessity to reach to an operational implementation of the ethical concept of Sustainable Development as proposed by the World Commission on Environment and Development [17]. Understanding of the major processes in the Earth system, and its changes over time, is one of the many prerequisites required for this operationalization, which depends on both the capability to monitor the state of the Earth system, and the capability to predict the future trajectories of the system under various assumptions. Both capabilities cannot be developed without a comprehensive monitoring of the Earth system. Recognizing the urgent need for a coordinated and sustained program of Earth observation, the recent Earth Observation Summits (EOS) have tasked the intergovernmental Group on Earth Observations (GEO) with the implementation of the Global Earth Observation System of Systems (GEOSS). GEOSS is designed with a focus on nine Societal Benefit Areas (SBAs) [6], including the SBAs of Climate, Weather, and Disasters.

Geodesy provides the foundation on which most Earth observation systems are built. In this function, the geodetic observing system is essential for Earth observation in general and GEOSS in particular. Geodesy is the science of measuring and mapping the geometry, rotation and gravity field of the Earth including their variations with time. These three characteristics of the Earth system are inherently related to the dynamics of the system, and to mass and energy transport throughout the system.

The development of space-geodetic technologies has revolutionized the methods of geodesy, and make possible the comprehensive monitoring of physical characteristics of the Earth system with high accuracy and resolution. Since the advent of space-geodesy about thirty years ago, the accuracy of positioning in a global geodetic reference frame has increased by roughly an order of magnitude every decade, reaching today sub-centimetre for relative positions on global scales and sub-millimetre per year changes in these positions. With today's spacegeodetic techniques, changes in the surface of the solid Earth, the oceans, land surface waters, and the ice sheets, can be measured with unprecedented accuracy and with ever increasing spatial and temporal resolution. These geodetic observations provide critical information on geodynamic processes underlying geohazards such as earthquakes, volcano eruptions, landslides, and subsidence, as well as changes in the global water cycle such as melting of ice sheets, sea level rise, and changes in land water storage. Variations in Earth rotation are inherently related to the global dynamics of the coupled atmosphere-ocean-solid Earth system. For observations of the time-variable Earth rotation, accuracy has also increased several orders of magnitude over the last few decades. These observations are not only critical for our understanding of the processes in the core and mantle of the solid Earth but also provide important constraints on large-scale climate phenomena and climate models. Dedicated gravity satellite missions have improved our quantitative knowledge of Earth's static gravity field to a level where they now provide vital constraints for ocean circulation models. Satellite observations of the time-variable gravity field, for the first time, provide accurate estimates of the changes in water storage on subcontinental scales and with temporal resolutions reaching ten days. The combination of the geodetic observations has allowed the determination of a global terrestrial reference frame with centimetre accuracy, an internal precision at the sub-centimetre level, and a longterm stability on the order of $1 \mathrm{~mm} / \mathrm{yr}$ or better.

Most of the technological developments in space-geodesy, and the rapidly improved scientific understanding of the observations, have been facilitated by the scientific expertise of the global geodetic community gathered in the International Association of Geodesy (IAG). Over the last fifteen years, the coordination of the global geodetic infrastructure, data collection and processing, and product development has come to a large extent through a number of technique- and product-specific IAG Services. These today provide valuable observations and products not only to the science community but also for a wide range of non-scientific applications. The International GNSS Service (IGS), established in 1994, provided the prototype model for these services. Recognizing the need to have a common voice for the increasing number of IAG Services as well as a link between IAG as a whole and relevant international Earth observation and research programs, the IAG initiated the Global Geodetic Observing System (GGOS) during the IUGG meeting in 2003 as an IAG Project. After an 
initial definition phase and the implementation of core elements of the organizational structure of GGOS, at the IUGG meeting in 2007 the IAG elevated GGOS to the status of permanent observing system of IAG.

The acronym "GGOS" has two very distinct aspects, which should be clearly distinguished: (1) the "organization GGOS", which consists of committees, panels, bureaus, and working groups, and (2) the "observation system GGOS", which comprises the infrastructure of a wide range of instrument types, satellite missions, and data and analysis centres. While GGOS as an organization has established its structure from essentially new entities, the infrastructure for GGOS is provided to a large extent by the existing IAG Services. The organizational components of GGOS are an integral part of the IAG structure (see, for example, [2] for a description of GGOS as an organization). With respect to the physical observing system, GGOS is facing a rather different situation: a considerable part of the infrastructure is provided by organizations that are not formally (or only loosely) affiliated with the IAG. The GPS system, for example, which is crucial for the IGS, is owned and maintained by the U.S. Department of Defense. Satellite missions contributing to GGOS, including satellite altimetry, satellite gravimetry, and InSAR, are implemented and operated by space agencies, while GGOS only utilizes the observations. Dedicated infrastructure such as the stations in the global tracking networks, the analysis centres, and even the components of the service organizations are provided on a voluntary basis. Consequently GGOS as an observing system depends crucially on infrastructure provided by others, and GGOS as an organization faces the challenge of ensuring, through dialogue with the relevant providers, that this infrastructure is available on a continuous basis. Considering the fact that the geodetic reference frames, which are made available through the IAG Services and GGOS, provide the backbone for geospatial services that underpin many modern societal applications, GGOS thus faces the challenge of promoting a core, but often invisible, element of the infrastructure of a modern society to those who, on the one hand, can provide the resources to sustain this infrastructure, and, on the other hand, can benefit from the geodetic observations and products.

\section{GEODESY'S CONTRIBUTION TO EARTH OBSERVATION}

Global geodesy serves Earth observation and global change science in two distinct ways: (1) geodesy provides the reference frames required for all location-dependent observations and thus contributes to the foundation of most Earth observations, and (2) geodesy provides observations of the time-variable shape, gravity field, and rotation of the Earth, and thus contributes to the Earth observation database. Within geodesy, the observations and the reference frames are inherently coupled. In fact, since the solid Earth is constantly deformed by internal geodynamic processes and by mass and energy transport in the fluid envelope of the solid Earth, the capability to understand and model these processes ultimately determines the accuracy of the global geodetic reference frames.

\subsection{The global geodetic reference frames}

Assigning time-dependent coordinates to points and objects, and describing the motion of the Earth in space requires access to an appropriate reference system. In geodesy, two global reference systems are basic for this purpose, namely the celestial reference system and the terrestrial reference system, which are dynamically linked to each other by the Earth's rotational motion. The two most accurate reference systems currently available are the International Celestial Reference System (ICRS) and the International Terrestrial Reference System (ITRS), which are conventional coordinate systems including all conventions for origin and orientation of the axes, scale, and physical constants, models, and processes to be used in their realization. These two systems are realized through their corresponding "reference frames," i.e., the International Celestial Reference Frame (ICRF; a set of estimated positions of extragalactic reference radio sources) and the International Terrestrial Reference Frame (ITRF; a set of estimated positions and velocities of globally distributed reference marks on the solid Earth's surface), respectively. These two frames are linked to each other by estimates of the Earth Orientation Parameters (EOPs). Based on the observations and analysis results provided by the IAG Services, the International Earth Rotation and Reference Systems Service (IERS) determines and provides access to the ITRF, the ICRF, and the EOPs.

The ITRF is today the most accurate realization of a global geodetic reference system. This well-defined, longterm stable, highly accurate and easily accessible reference frame is the basis for all precise positioning on and near the Earth's surface. The ITRF underpins all geo-referenced data used by society for so many uses, and it is the basis for most other reference frames, including WGS84 and the reference frame GTRF for the GALILEO 
system. Because of this, the ITRS and the ITRF are not only indispensable for Earth observation in general, but also for many societal applications ranging from navigation, mapping, surveying, national and regional reference frames, to civil engineering.

\subsection{Role of geodetic observations for science}

As mentioned above, geodesy is the science of determining the geometry, gravity field, and rotation of the Earth, and their evolution in time. Modern geodetic techniques permit the measurement of changes in the geometry of the Earth's surface with an accuracy of millimetres over distances of several $1000 \mathrm{~km}$. The secular surface kinematics can be monitored with sub-mm/yr accuracy. Geodetic imaging techniques increasingly gain importance, particularly when integrated with the traditional point-based approach of geodesy. Based on these geometric techniques, temporal changes in the Earth's shape, rotation and gravity field are provided with increasing spatial and temporal resolution, increasing accuracy, and with decreasing latency. The internationally coordinated global geodetic station networks provide a continuous monitoring of the ITRF and the EOPs. In combination with ITRF, the Global Navigation Satellite Systems (GNSS) provide access to precise point coordinates in ITRF anytime and anywhere on the Earth's surface with centimetre-level accuracy.

Data from a large number of space missions and other geodetic observations have contributed to the determination of models of the Earth's gravity field. The significant improvement in the resolution and precision of our knowledge of the Earth's gravity field is due to the satellite missions CHAMP and GRACE, in orbit since 2000 and 2002, respectively. In particular GRACE has pushed our knowledge of the static geoid to centimetrelevel accuracy. The European GOCE mission, launched on 17 March 2009, will further improve precision and resolution of the static part of the gravity field to an unprecedented level. The GRACE mission is designed to monitor changes in Earth's gravity field induced by mass changes at spatial scales less than $500 \mathrm{~km}$ and with amplitudes of the order of $1 \mathrm{~cm}$ of water equivalent.

All these geodetic observations record the "fingerprints" of mass movements in the solid Earth, ocean, atmosphere, ice sheets and terrestrial water storage; they allow the determination of the displacement field associated with earthquakes and the velocity and strain fields of the Earth's surface; they scale mass and volume changes in the ocean; they monitor the changes in ice sheets; they sense the changes in land water storage; they measure the water content in the atmosphere; and they provide crucial constraints for all models of geophysical processes in the Earth system.

At the accuracy level reached today in space-geodesy, the variations in the Earth's shape, gravity field, and rotation are caused by a number of Earth system processes acting on a wide range of spatial and temporal scales. Meaningful interpretation of the geodetic observations therefore requires the development of models that can predict the geodetic observations for an increasing number of these processes with the same accuracy as, or better than, the observations. In the course of this process, geodesy has developed into a science making unique contributions to the understanding of the Earth system, its dynamics, and its response to climate change. Consequently, with modern instrumentation and analytical techniques, the scope of geodesy is rapidly extending from a mere monitoring of the geometric, gravitational and rotational changes, to the modelling and understanding of the causes of the observed changes. With this broader scope, geodesy contributes not only to the scientific understanding of the Earth system but also the functioning, and security of society in general.

\section{GGOS: A MULTI-TECHNIQUE, MULTI-LAYERED YET INTEGRATED SYSTEM}

\subsection{A value-chain from observations to applications}

GGOS provides products that are pivotal for Earth observation, Earth science, geo-information systems, and terrestrial and planetary navigation. From a value-chain point of view, connecting observations at one end of the chain to user applications at the other end, GGOS comprises four main components. (1) The instrumentation includes global terrestrial networks of geodetic stations and observatories, Earth observing satellites, satellite navigation systems, and planetary missions. (2) The data infrastructure comprises the infrastructure for data transfer, data management and archiving, and data and product dissemination. (3) The data analysis covers the complete and consistent data processing chain from the initial acquisition and the processing of large amounts of observations, to the consistent integrated analysis and combination of products, and the assimilation of the 
observations into complex models of the Earth system or components of this system. (4) The GGOS Portal provides a unique access point for GGOS users to all GGOS products, including relevant metadata and documentation

GGOS faces the challenge to develop the geodetic technologies and the observing system so that they meet the demanding and evolving observational requirements in terms of reference frame accuracy and availability, as well as in terms of spatial and temporal resolution and accuracy of the geodetic observations. GGOS has assessed these requirements for science, Earth observations and societal application (see [7]). The most demanding observational requirements result mostly from scientific applications. For reference frame accuracy, scientific studies of sea level change caused by climate change appear to pose the most demanding requirements of accuracy and stability at the levels of $1 \mathrm{~mm}$ and $0.1 \mathrm{~mm} / \mathrm{yr}$, respectively. For the geoid, for use in ocean general circulation models to define the mean sea surface topography, and the use with GNSS for the determination of heights at the millimetre level requires the static geoid to be accurate and stable at levels of 1 $\mathrm{mm}$ and $0.1 \mathrm{~mm} / \mathrm{yr}$, respectively. For Earth orientation parameters, the most demanding application is likely to be the tracking and navigation of interplanetary spacecraft, which is a capability-driven application requiring the most accurate EOPs that can be determined, such that they are consistent in accuracy with the accuracy of the ITRF and ICRF.

\subsection{A system-of-systems}

From a system-oriented point of view, the global geodetic infrastructure can be characterized as a multitechnique "system-of-systems", which observes key geometric and physical quantities of the Earth system with specialized technologies (Table 1). The technique-specific IAG Services take care of the coordination of infrastructure relevant for a specific technique. Coordination between techniques and combination of products from different techniques to higher-level products is undertaken by the IERS and the International Gravity Field Service (IGFS).

Table 1: The Global Geodetic Observing System (GGOS). VLBI: Very Long Baseline Interferometry; SLR: Satellite Laser Ranging; LLR: Lunar Laser Ranging; GNSS: Global Navigation Satellite Systems; DORIS: Doppler Orbitography and Radio positioning Integrated by Satellite; InSAR: Interferometric Synthetic Aperture Radar; IGS: International GNSS Service; IAS: International Altimetry Service; IVS: International VLBI Service for Geodesy and Astrometry; ILRS: International Laser Ranging Service; IDS: International DORIS Service; IERS: International Earth Rotation and Reference Systems Service; IGFS: International Gravity Field Service; GGP: Global Geodynamics Project; BGI: International Gravimetric Bureau; IgeS: International Geoid Service.. Modified from [12].

\begin{tabular}{|l|l|l|l|}
\hline Component & Objective & Techniques & Responsibility \\
\hline $\begin{array}{l}\text { I. Geokinematics } \\
\text { (size, shape, } \\
\text { kinematics, } \\
\text { deformation) }\end{array}$ & $\begin{array}{l}\text { Shape and temporal variations of } \\
\text { land/ice/ocean surface (plates, intra- } \\
\text { plates, volcanos, earthquakes, glaciers, } \\
\text { ocean variability, sea level) }\end{array}$ & $\begin{array}{l}\text { altimetry, InSAR, GNSS- } \\
\text { cluster, VLBI, SLR, DORIS, } \\
\text { imaging techniques, levelling, } \\
\text { tide gauges }\end{array}$ & $\begin{array}{l}\text { International and national } \\
\text { projects, space missions, IGS, } \\
\text { IAS, future InSAR service }\end{array}$ \\
\hline $\begin{array}{l}\text { II. Earth Rotation } \\
\text { (nutation, precession, } \\
\text { polar motion, } \\
\text { variations in length- } \\
\text { of-day) }\end{array}$ & $\begin{array}{l}\text { Integrated effect of changes in angular } \\
\text { tementum and moment of inertia } \\
\text { tensor (mass changes in atmosphere, } \\
\text { cryosphere, oceans, solid Earth, } \\
\text { core/mantle; momentum exchange } \\
\text { between Earth system components) }\end{array}$ & $\begin{array}{l}\text { classical astronomy, VLBI, } \\
\text { LLR, SLR, GNSS, DORIS, } \\
\text { under development: terrestrial } \\
\text { gyroscopes }\end{array}$ & $\begin{array}{l}\text { International geodetic and } \\
\text { astronomical community (IERS, } \\
\text { IGS, IVS, ILRS, IDS) }\end{array}$ \\
\hline III. Gravity field & $\begin{array}{l}\text { Geoid, Earth's static gravitational } \\
\text { potential, temporal variations induced } \\
\text { by solid Earth processes and mass } \\
\text { transport in the global water cycle. }\end{array}$ & $\begin{array}{l}\text { Terrestrial gravimetry (absolute } \\
\text { and relative), airborne } \\
\text { gravimetry, satellite orbits, } \\
\text { dedicated satellite missions } \\
\text { (CHAMP, GRACE, GOCE) }\end{array}$ & $\begin{array}{l}\text { International geophysical and } \\
\text { geodetic community (GGP, } \\
\text { IGFS, IGeS, BGI) }\end{array}$ \\
\hline IV. Terrestrial Frame & $\begin{array}{l}\text { Global cluster of fiducial points, } \\
\text { determined at mm to cm level }\end{array}$ & $\begin{array}{l}\text { VLBI, GNSS, SLR, LLR, } \\
\text { DORIS, time keeping/transfer, } \\
\text { absolute gravimetry, gravity } \\
\text { recording }\end{array}$ & $\begin{array}{l}\text { International geodetic community } \\
\text { (IERS with support of IVS, ILRS, } \\
\text { IGS, and IDS) }\end{array}$ \\
\hline
\end{tabular}




\subsection{A multi-layered system}

Looking from the Earth's surface upward, the global geodetic infrastructure appears to be layered and can be characterized by five major levels of instrumentation and objects that actively perform observations, are passively observed, or both (Figure 1). These levels are [13]:

Level 1: the terrestrial geodetic infrastructure, which comprises ground stations of in situ and tracking networks, observatories, communication links, and infrastructure for data storage, processing, modelling, and distribution;

Level 2: the Low-Earth Orbiting (LEO) satellite missions, which include different types of altimeters, synthetic aperture radar, gravity satellite missions, LIght Detection And Ranging (LIDAR) sensors, and other instruments; Level 3: the satellites of the Global Navigation Satellite Systems (GNSS) and the LAGEOS-type Satellite Laser Ranging (SLR) satellites;

Level 4: the planetary missions and geodetic infrastructure on Moon and planets, including reflectors for Lunar Laser Ranging (LLR);

Level 5: the extragalactic radio-signal emitting quasars.

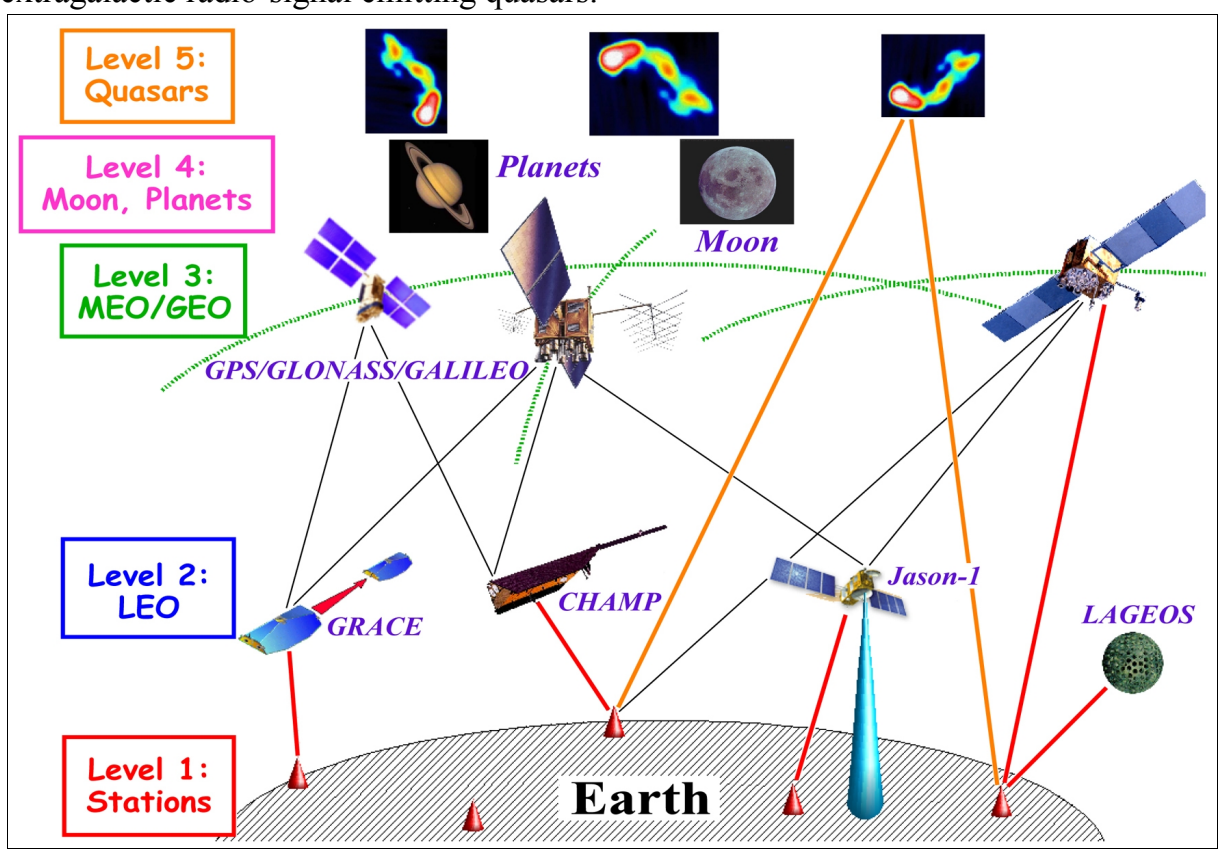

Fig. 1. The five layers of the global geodetic infrastructure. From [13].

\subsection{Integration through multiple links}

Independent of whether the instruments and objects in a layer are active or passive, receivers or emitters or both, these five levels of instrumentation and objects are connected in various ways. Firstly, all geodetic techniques measure the "output" of the same unique Earth system and are thus affected by the same environmental processes, although with different sensitivities and noise sources. Most, if not all, physical processes in the Earth system are associated with mass redistributions (see Section 4). The geodetic fingerprints of these mass redistributions and changes in the system's dynamics are captured by the different techniques according to their specific sensitivity. For example, displacements of the surface of the solid Earth, oceans, ice sheets, and land water areas are observed with several independent geometric and imaging techniques. Earth rotation variations are derived from several independent techniques and through combinations of these techniques; and changes in surface mass storage are extracted from observations of the time-variable gravity field, Earth rotation, and surface displacements. Thus, the different parts of the overall system are cross-linked through observations of the same geometric and physical quantities, and, to a certain extent, they are inter-dependent. In the data processing, the geodetic observations are further linked through geophysical models of the system processes. Therefore, consistency of data processing, modelling, and conventions across the techniques and across the three main areas of geodesy is mandatory if the full potential of the observing system is to be realized. 
Most importantly, for the integration of the individual layers and systems into a consistent observing system, the different techniques are connected through co-location of sensors both on Earth and in space. For the separation of technique-dependent effects from the fingerprints of the Earth system processes in the geodetic observations, co-location of different and often complementary techniques is crucial. Co-location of different techniques at the same location on Earth has vital for ensuring the stability of the ITRF. Today, the uneven geographical distribution of "core" stations with three or more space-geodetic techniques is a limiting factor for the accuracy of the ITRF. GGOS must work towards a more even distribution of core stations.

The emerging co-location of different sensors and observation types onboard a satellite is also extremely important to establish connections between the observation techniques. This in-space co-location complements the co-location of stations on the Earth. An example is the rapid progress achieved in orbit determination with the tracking data of the TOPEX/Poseidon satellite using DORIS, GPS, SLR and altimetry crossovers. GGOS is therefore promoting that future satellite missions put emphasis on establishing links between different observation and tracking techniques. For example, it is of particular importance that all GNSS satellites be equipped with laser retro-reflectors arrays, thus providing a link between GNSS and SLR.

\subsection{An integrated system sensing atmosphere, hydrosphere, and solid Earth}

Facilitated by these links through physics, models and co-location, the five layers of the global geodetic infrastructure form the complex, integrated GGOS as an observing system. The major observation types acquired by this system include: (1) observations of the microwaves emitted by GNSS satellites at the ground stations and at LEO satellites; (2) laser ranging to LEOs, dedicated laser ranging satellites, GNSS satellites and the Moon; (3) observation of the microwaves emitted by quasars using antennas in the Very Long Baseline Interferometry (VLBI) network; (4) instrumentation onboard the LEO satellites measuring accelerations, gravity gradients, satellite orientation, etc.; (5) radar and optical observations of the Earth's surface (land, ice, glaciers, sea level, ect.) from remote sensing satellites; (6) distance measurements between satellites (K-band, optical, interferometry, etc.); (7) measurements of other quantities on the ground related to variations in gravity (with absolute and relative gravimeters), Earth rotation (for example, with ring lasers), sea level (for example, with tide gauges), and water storage (for example, lake groundwater levels) (see [13] for more details). For many of these observations, in particular, the space-geodetic observations, time measurements are crucial and, ultimately, the accuracy of the geodetic observations depends on the accuracy of time measurements.

With these observations, the geodetic infrastructure not only measures time-variable Earth shape, gravity field, and rotation. As they propagate through the ionosphere and troposphere, the microwaves are refracted by the atmosphere and thus sense this component of the Earth system as well.

\section{GLOBAL CHANGE RESULTS}

Many of the burning questions related to the water cycle, climate variability, global change, and geohazards cannot be solved without knowledge of energy and mass transports throughout 'System Earth' and the associated dynamics. Most of the processes leading to mass and energy transport affect the Earth's figure (geometry), its gravity field and its rotation. Consequently, geodetic observations record the fingerprints of global change processes. However, the signals induced by global change in geodetic observations are generally small (of the order of parts-per-billion of the quantities), and they are often embedded in larger variations not caused by global change. Therefore, in addition to geodetic observations with an accuracy considerably better than these signals, identifying and quantifying the global change signals also requires accurate modelling of all known processes in an Earth system model.

Many scientific studies of global processes, including those related to global and climate change, have already benefited from detailed knowledge of the Earth's time-variable shape, rotation, and gravity field. Over the last fifteen or so years, the global geodetic networks have provided an increasingly detailed picture of the temporal variations in the Earth's shape and of the kinematics of points on the Earth's surface, including the ocean, ice cover, and land surfaces. The observations have been used, for example, to determine improved models of the secular velocity field as an input for studies of plate tectonics, post-glacial rebound, sea level changes, and ice load changes, to derive models of the global strain rate field (Figure 2), to study seasonal loading and derive seasonal variations in the terrestrial hydrosphere, to invert for mass changes in ocean, ice sheets and land water 
storage, to improve the modelling of the seasonal term in polar motion, and to study transient surface deformations prior to, during and after earthquakes. Geodetic techniques provide the means to observe surface deformations on volcanoes, in unstable landslide prone areas, associated with earthquakes and fault motion, or subsidence caused by anthropogenic activities such as groundwater and oil extraction (for more examples and references, see [11]). In the near future, geodetic observing techniques will be able to determine the magnitude and displacement field of great earthquakes in near real-time as support for early warning systems (e.g., [4]).

Variations in Earth's rotation are induced by mass transport in the Earth system and the exchange of angular momentum among its components. Earth rotation observations have provided insight into many global-scale dynamic phenomena, including sea level changes, post-glacial rebound, and hemispheric seasonal changes in land water storage and snow load. An example at interannual time scales is the El Niño/Southern Oscillation (ENSO) phenomenon, which is the most prominent feature of the climate system at these time scales. ENSO event are associated with a collapse of the tropical easterlies leading to an increase of the atmospheric angular momentum, which in turn is compensated for by a decrease of the solid Earth's angular momentum and an increase of the length-of-day of up to 0.5 milliseconds (for particularly strong ENSO events). ENSO events are associated with significant and far-reaching impacts on interannual climate, and early detection and forecasting of the timing and magnitude of these events is of high societal relevance. Geodetic observations of Earth rotation and sea surface height anomalies provide pivotal constraints for both detection and forecasting (for references, see [14]).

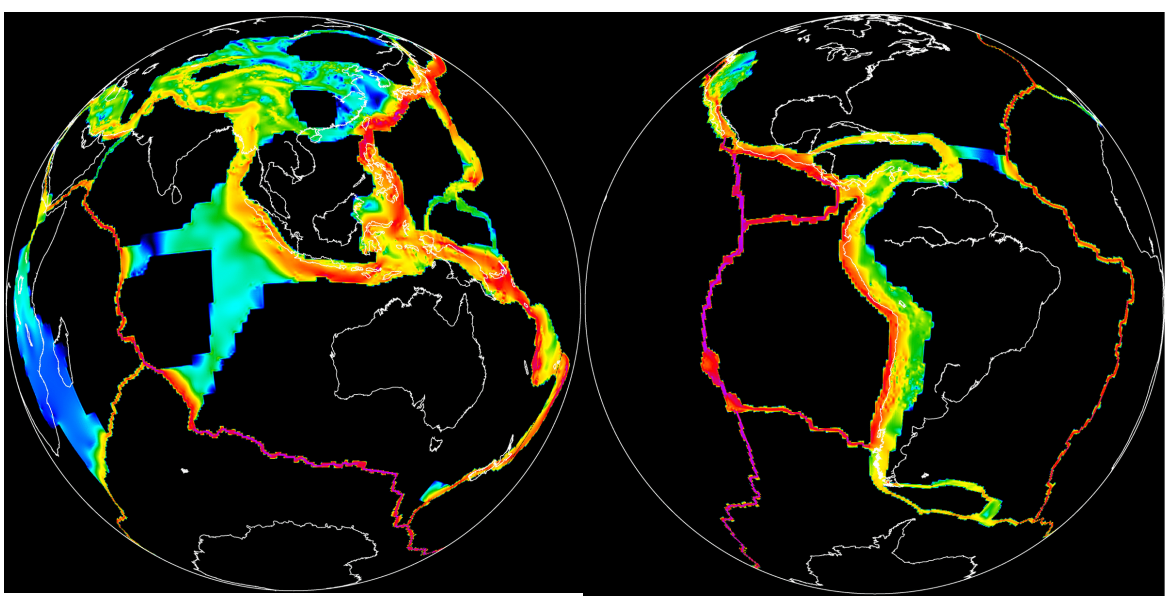

Fig. 2. Geodetic observations of Earth's time-variable shape are used, for example, to determine strain rate models. Shown is the second invariant of a strain rate tensor model obtained from global GPS observations. Courtesy C. Kreemer.

Gravity field variations are caused by many different processes in the Earth system (Figure 3), and most of these processes have geodetic fingerprints above the accuracy level reached by space-geodesy. For example, gravity effects of co-seismic and post-seismic deformation associated with large earthquakes have been identified in GRACE observations (e.g., [1],[10]). At time scales from weeks to several decades, the largest mass redistributions on the surface of the solid Earth occur in the hydrological cycle, and the GRACE mission is providing unprecedented insight into the water cycle down to spatial scales of about $500 \mathrm{~km}$ and on sub-monthly time scales (Figure 4; for references, see [12]).

Figure 3 illustrates that the different processes are associated with different temporal and spatial scales, which lead to multiple geodetic signatures. Mass movements in the hydrosphere have temporal scales from weeks to decades, and spatial scales from $10 \mathrm{~km}$ to continental or basin scales. These mass movements load and deform the solid Earth, impact on the gravity field of the Earth system, and change the rotation of the solid Earth. The response of the solid Earth to the loads includes an elastic response to concurrent mass redistributions, as well as a viscous response to past mass movements. The response in Earth's shape, gravity field and rotation depends on the spatial scale of the loads, with surface displacements being more sensitive to smaller loads than gravity and rotation. Consequently, the different geodetic techniques have specific temporal and spatial sensitivities. Sampling of the complete spatio-temporal spectrum with high resolution and accuracy with a set of different techniques is therefore necessary in order to be able to invert the geodetic observations to derive mass changes 
associated with the individual Earth system processes. For example, inversion of surface displacements observed with GPS and geophysical models of these displacements have errors at the level of a few centimetres at a few hundred $\mathrm{km}$ wavelength, while mass estimates derived from GRACE at $200 \mathrm{~km}$ have errors larger than $20 \mathrm{~cm}$. A combination of integrated geodetic observations with models of the water cycle is therefore a promising approach. For example, results of combined inversion of observations from GRACE, SLR, VLBI, GPS and predictions of an ocean model (ECCO) show a dramatic melting of Greenland (Wu, 2008, personal communication).

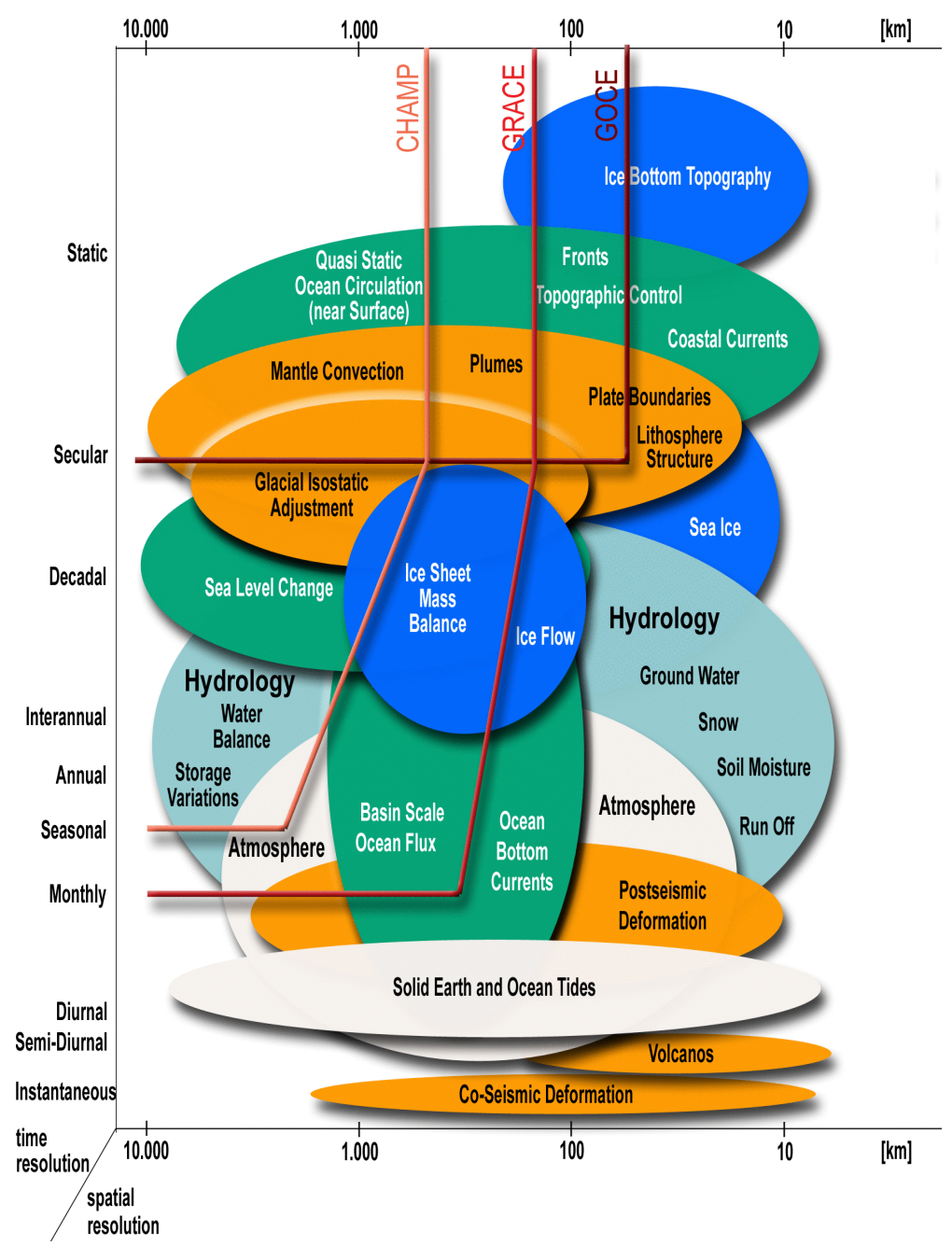

Fig. 3. Mass transport in the Earth system. The different processes are associated with characteristic spatial scales, ranging from a few $\mathrm{km}$ to global, and tenporal scales from instantaneous to secular and static. The capability of current dedicated gravity satellite missions to capture the "fingerprint" of some of these processes is indicated. From [9].

There are many climate-related scientific problems that can be addressed using combined geodetic observations. For example, one of the projects in the Global Energy and Water Cycle Experiment (GEWEX) addresses the question of how changes in land use and aerosol may have impacted monsoons (Lawford, 2008, personnel communication). Geodetic observations can provide important constraints on the associated changes in land water storage. Other areas are extremes: there are periods of 'quiet' variations and then there are periods of extreme variations, and geodetic observations may help to characterize and potentially predict these periods. For example, the forecasts of the drought conditions in 2005 in Canada failed completely (Lawford, 2008, personnel communication), but using global geodetic observations to characterize the state of the Earth system may help to improve such forecasts. There is also an urgent need to improve season-by-season prediction. On the one hand, geodetic observation support the validation of numerical (weather and climate) prediction models, and, on the 
other hand, assimilation of these observations into prediction models may in the future improve the predictive capabilities of these models.
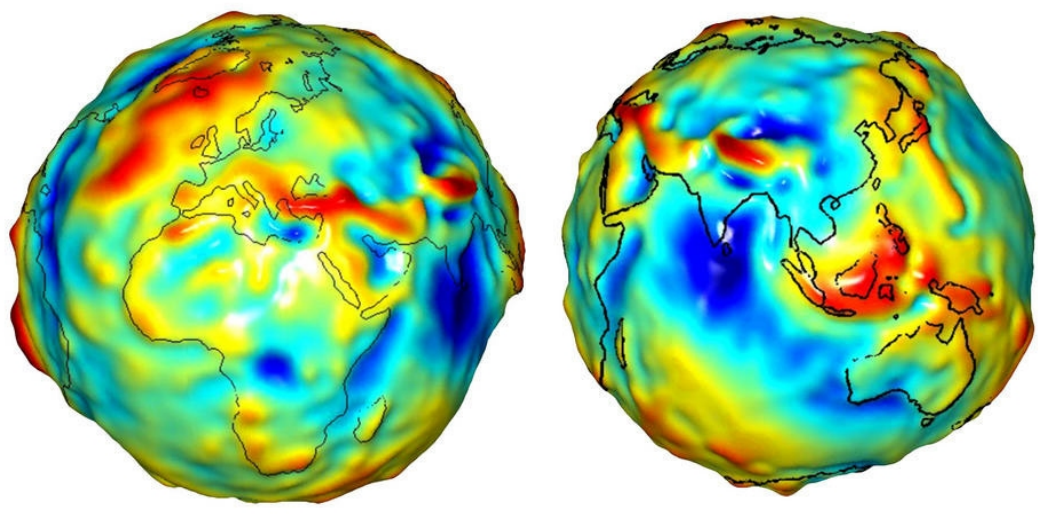

Fig. 4. Geodetic observations of the time-variable gravity field of the Earth. The pictures show a snapshot of monthly changes in the Earth's gravity field with respect to the mean field. The changes are caused mainly by mass transport in the fluid envelope of the solid Earth. Courtesy NASA.

Combination of the full suite of geodetic observations with land surface hydrological models helps to increase spatial resolution. An important advantage of the geodetic observations is that they are not limited in terms of penetration depth: subsurface mass changes have their fingerprint in all geodetic observations. Current challenges for an operational use of the geodetic observations, for example, in water management, drought and seasonal predictions, include considerable differences between observed and modelled variables, a spatial resolution of observations that is too coarse, a temporal resolution that is too low, and a latency that is too high.

The gravity field determination from dedicated space missions has also contributed tremendously to advances in a number of fields of geodesy, including reference frames, the orbits of ocean radar altimetry satellites, laser altimeters, and sea surface topography from satellite altimetry. The integration of various satellite missions with the geometric techniques created new opportunities for the study of mass transport in the Earth system in a globally consistent way. Oceanographic applications illustrate the unique way in which the combined geodetic observations provide accurate and quantitative constraints on the ocean mass budget, tidal dissipation, nearsurface ocean flow and its variability, and large-scale ocean mass variations. The observations are invaluable for understanding the causes of sea level rise and the dynamics of ocean mass redistribution. Geodetic observations are also crucial in reducing the current large uncertainties in future global and local sea level predictions, a key issue hampering the development of urgently needed adaptation policies for one of the most severe impacts of global warming: coastal inundation.

In order to fully exploit the complimentary nature of the changes in Earth's shape, gravity field and rotation for global change studies, it will be necessary to develop the observing system based on our understanding of the geodetic fingerprints of these changes. However, currently other factors have a greater impact on the development of geodetic infrastructure. As an example, we compare areas of large on-going mass changes to the network of GPS stations. The secular trends in surface mass as determined from GRACE is shown in Figure 5. These mass changes induce both gravity changes and surface displacements, with the ratio of surface displacements to gravity changes increasing with decreasing spatial scales. Therefore, observations of surface displacements, particularly for areas where GRACE senses large secular mass variations, would be complementary to the gravity observations. However, comparing the locations of GPS stations for which observations are available in public data archives, it is obvious that only some areas with large mass decreases are well covered with GPS stations (e.g., North America), while others are not (e.g., Greenland, Africa, South America, and Antarctica, see Figure 5). Future deployments of GNSS sites should therefore aim to reduce the mismatch between areas with mass changes and GNSS coverage. 
determination and for radio occultation studies, star sensors for attitude, an SLR retro-reflector and a VLBI signal transmitter. As the satellite (or satellite constellation) orbits the Earth it would "connect" all the global core sites and improve co-location from space.

Currently, temporal inhomogeneities of geodetic time series due to technological developments, changing station distribution, and improvements in geophysical models and geodetic analysis methods are a limitation for many global change studies. Developments in analysis capabilities through new approaches for single techniques (such as, for example, the recent ambizap algorithm, see [3]) or combined analyses, improvements of the reference frame (for example, [8]) and the geophysical models, as well as increased computer storage and processing power will provide the necessary capabilities for rapid and homogeneous reprocessing of the complete global geodetic database with the highest possible accuracy in order to provide datasets for global change and climate studies that are as homogeneous in time as possible. A prerequisite for exploiting the full potential of geodesy for Earth observation, Earth system monitoring, and for many practical applications is, however, a sophisticated integration of all geodetic techniques (spaceborne, airborne, marine and terrestrial), processing models and geophysical background models into one system. This integration will permit - as part of global change research - the assessment of surface deformation processes and the quantification of mass anomalies and mass transport inside individual components, and mass exchange between the components of the Earth's system. GGOS is pivotal in facilitating this integration.

\section{ACKNOWLEDGEMENTS}

The authors are grateful to the IAG Community, the Services, Commissions, the GGOS Steering and Executive Committees, the Science Panel, and the Working groups: GGOS is built by the best effort of the many individuals in these IAG and GGOS components. GGOS also depends on the continuous support of many other contributors, in particular the space agencies, which provide infrastructure crucial to GGOS. The GPS site locations for the maps in Figure 5 were provided by Corne Kreemer and Geoff Blewitt. Part of the work carried out by the lead author was supported by several NASA grants under the ROSES program and by a JPL contract.

\section{REFERENCES}

[1] Bao, L.F., Piatanesi, A., Lu, Y., Hus, H.T., \& Zhou, X.H., 2005. Sumatra tsunami affects observations by GRACE satellites, Eos, Trans. Am. Geophys. Union, 86, 353,356.

[3] Beutler, B., Pearlman, M., Plag, H.-P., Neilan, R., Rothacher, M., \& Rummel, R., 2008. Towards GGOS in 2020, in The Global Geodetic Observing System: Meeting the Requirements of a Global Society on a Changing Planet in 2020, edited by H.-P. Plag \& M. Pearlman, Springer Verlag, Berlin, in press.

[5] Blewitt, G., 2008. Fixed-point theorems of GPS carrier phase ambiguity resolution and their application to massive network processing: 'Ambizap', J. Geophys. Res., 113, B12410, doi: $10.1029 / 2008$ JB005736.

[7] Blewitt, G., Kreemer, C., Hammond, W., Plag, H.-P., Stein, S., \& Okal, E., 2006. Rapid determination of earthquake magnitude using GPS for tsunami warning systems, Geophys. Res. Lett., 33, L11309, doi:10.1029/2006GL026145.

[9] Doran, P.T. \& Kendall, M., 2009. Examining the scientific consensus on climate change, Eos, Trans. Am. Geophys. Union, 90, 22-23.

[10]

[11] GEO, 2005. Global Earth Observing System of Systems GEOSS - 10-Year Implementation Plan Reference Document - Draft\}, Tech. Rep. GEO 1000R/ESA SP 1284, ESA Publication Division, ESTEC, Noordwijk, The Netherlands, Avaliable at http://earthobservations.org.

[12]

[13] Gross, R., Beutler, G., \& Plag, H.-P., 2009. Integrated scientific and societal user requirements and functional specifications for the GGOS, in The Global Geodetic Observing System: Meeting 
the Requirements of a Global Society on a Changing Planet in 2020, edited by H.-P. Plag \& M.

[15] Herring, T.A., Altamimi, Z., Plag, H.-P., \& Poli, P., 2009. The future geodetic reference frame, in The Global Geodetic Observing System: Meeting the Requirements of a Global Society on a [16] Changing Planet in 2020, edited by H.-P. Plag \& M. Pearlman, Springer Verlag, Berlin, in press.

[17] Ilk, K.H., Flury, J., Rummel, R., Schwintzer, P., Bosch, W., Haas, C., Schroeter, J., Stammer, D., Zahel, W., Miller, H., Dietrich, R., Huybrechts, P., Schmeling, H., Wolf, D., Goetze, H.J., Riegger, J., Bardossy, A., Guenter, A., \& Gruber, T., 2005. Mass transport and mass distribution in the Earth system, Tech. rep., GOCE-Projectbuero Deutschland, Technische Universitaet Muenchen, GeoForschungsZentrum Potsdam.

[19] Panet, I., Mikhailov, V., Politz, F., \& Diament, M., 2008. Insight into the Sumatra December 2004 and March 2005 post-seismic signals from GRACE gravity variations, in Proceedings of the GRACE Science Team Meeting, December 2008, edited by J. Ries \& S.Bettadpur, 432-438.

[21] Plag, H.-P., Beutler, G., Gross, R., Herring, T.A., Rizos, C., Rothacher, M., Rummel, R., Sahagian, D., \& Zumberge, J., 2009a. Introduction, in The Global Geodetic Observing System: Meeting the Requirements of a Global Society on a Changing Planet in 2020, edited by H.-P.

[25] Rothacher, M., Beutler, G., Bosch, W., Donnellan, A., Gross, R., Hinderer, J., Ma, C., Pearlman, M., Plag, H.-P., Ries, J., Schuh, H., Seitz, F., Shum, C.K., Smith, D., Thomas, M., Velacognia, E., Wahr, J., Willis, P., \& Woodworth, P.L., 2008. The future Global Geodetic Observing System (GGOS), in The Global Geodetic Observing System: Meeting the Requirements of a Global Society on a Changing Planet in 2020, edited by H.-P. Plag \& M. Pearlman, Springer Verlag, Berlin, in press.

[27] Rummel, R., Beutler, B., Dehant, V., Gross, R., Ilk, K.H., Plag, H.-P., Poli, P., Rothacher, M., Stein, S., Thomas, R., Woodworth, P.L., Zerbini, S., \& Zlotnicki, V., 2009. Understanding a dynamic planet: Earth science requirements for geodesy, in The Global Geodetic Observing System: Meeting the Requirements of a Global Society on a Changing Planet in 2020, edited by H.-P. Plag \& M. Pearlman, Springer Verlag, Berlin, in press.

[28] Turner II, B.L., Clark, W.C., Kates, R.W., Richards, J.F., Mathews, J.T., \& Meyer, W.B., eds., 1990. The Earth as Transformed by Human Action: Global and Regional Changes in the Biosphere Over the Past 300 Years, University Press, Cambridge, 713 pages.

[30] Watkins, M.M., Yuan, D.N., Kuang, D., Bertiger, W., Byun, S., Lu, W., \& Kruizinga, G.L., 2008. JPL L-2 GRACE solutions: Harmonics, mascons, iteration, and constraints, in Proceedings of the $[31]$ GRACE Science Team Meeting, December 2008, edited by J. Ries \& S. Bettadpur, 82-92.

[32] World Commission on Environment and Development, 1987. Our Common Future, Oxford University Press, Oxford. 